\title{
Comprehensive circular RNA profiling reveals the regulatory role of the hsa_circ_0137606/miR-1231 pathway in bladder cancer progression
}

\author{
WEIJIAN LI ${ }^{1 *}$, YOUJIAN LI ${ }^{1 *}$, ZHONGXU SUN $^{1 *}$, JUN ZHOU $^{1}$, YUEPENG CAO $^{1,2}$, \\ WENLIANG MA ${ }^{1}$, KAIPENG XIE ${ }^{3,4}$ and XIANG YAN ${ }^{1,5}$ \\ ${ }^{1}$ Department of Urology, Drum Tower Hospital, Medical School of Nanjing University, Institute of Urology, \\ Nanjing University, Nanjing, Jiangsu 210008; ${ }^{2}$ Department of Critical Care Medicine, Jiangsu Cancer Hospital, \\ Jiangsu Institute of Cancer Research, Nanjing Medical University Affiliated Cancer Hospital, Nanjing, \\ Jiangsu 210009; ${ }^{3}$ Nanjing Maternity and Child Health Care Institute, Women's Hospital of Nanjing Medical University; \\ ${ }^{4}$ Department of Women Health Care, The Affiliated Obstetrics and Gynecology Hospital of Nanjing Medical University, \\ Nanjing Maternity and Child Health Care Hospital, Nanjing, Jiangsu 210004; ${ }^{5}$ Department of Urology, \\ Anqing Petrochemical Hospital, Nanjing Gulou Hospital Group, Anqing, Anhui 246002, P.R. China
}

Received May 23, 2019; Accepted August 7, 2019

DOI: $10.3892 /$ ijmm.2019.4340

\begin{abstract}
Bladder cancer (BC) is one of the most common malignant tumors in males globally. Its progression imposes a heavy burden on patients; however, the expression profile of circular (circ)RNAs in BC progression remains unclear. This study explored changes in circRNA expression during $\mathrm{BC}$ progression by sequencing different grade $\mathrm{BC}$ samples and normal controls to reveal the circRNA expression profiles of different BC grades. Gene Ontology (GO) and Kyoto Encyclopedia of Gens and Genomes (KEGG) pathway analyses, and protein-protein interaction network construction
\end{abstract}

Correspondence to: Dr Xiang Yan, Department of Urology, Drum Tower Hospital, Medical School of Nanjing University, Institute of Urology, Nanjing University, 321 Zhongshan Road, Nanjing, Jiangsu 210008, P.R. China

E-mail: yanxiang01975@126.com

Dr Kaipeng Xie, Department of Women Health Care, The Affiliated Obstetrics and Gynecology Hospital of Nanjing Medical University, Nanjing Maternity and Child Health Care Hospital, 123 Tianfei Lane, Mochou Road, Nanjing, Jiangsu 210004, P.R. China

E-mail: xiekaip0110@yeah.net

*Contributed equally

Abbreviation: BC, bladder cancer; ceRNAs, competitive endogenous RNAs; PPI, protein-protein interaction; NMIBC, non-muscle-invasive bladder cancer; MIBC, muscle-invasive bladder cancer

Key words: circular RNA, progression, bladder cancer, hsa_circ_0137606, microRNA-1231 were used to predict pathways that the differentially expressed circRNAs may participate in. circRNA expression levels were detected using reverse transcription-quantitative polymerase chain reaction (RT-qPCR) and dual-luciferase reporter assays were used to investigate the interactions between circRNA and microRNA (miR). Cell Counting Kit-8 and Transwell assays were also performed to detect cell proliferation, migration, and invasion. In total, 244 circRNAs were found to be differentially expressed in high-grade $\mathrm{BC}$ compared to low-grade $\mathrm{BC}$, whilst 316 dysregulated circRNAs were detected in high-grade BC compared with normal urothelium. Furthermore, 42 circRNAs overlapped between the two groups, seven of which were randomly selected and detected by RT-qPCR to validate the sequencing results. GO analysis and KEGG pathway analyses revealed that the differentially expressed circRNAs may participate in BC via 'GTPase activity regulation', 'cell junction', and 'focal adhesion' pathways. Of note, we proposed that a novel circRNA in BC progression, hsa_circ_0137606, could suppress BC proliferation and metastasis by sponging miR-1231. Through bioinformatics analysis, we predicted that $\mathrm{PH}$ domain and leucine rich repeat protein phosphatase 2 could be a target of the hsa_circ_0137606/miR-1231 axis in $\mathrm{BC}$ progression. Using high-throughput sequencing, this study revealed the circRNA expression profiles of different grades of BC and proposed that the novel circRNA, hsa_circ_0137606, suppresses BC proliferation and metastasis by sponging miR-1231. Our findings may provide novel insight into potential therapeutic targets for treating BC.

\section{Introduction}

Bladder cancer (BC) is the ninth most common cancer globally, with an annual incidence of 430,000 cases $(1,2)$. It is a complex disease associated with high mortality rates without appropriate treatment (3). BC includes non-muscle-invasive 
BC (NMIBC) and muscle-invasive BC (MIBC); 10-15\% of patients with NMIBC will progress to MIBC (4). Currently, there is no ideal treatment for high-grade invasive BC. Radical cystectomy and chemotherapy are alternative treatment options; however, both significantly reduce patient quality of life and survival time $(5,6)$. Therefore, to prevent $\mathrm{BC}$ progression more effectively, the mechanisms of $\mathrm{BC}$ progression must be investigated.

Circular RNAs (circRNAs) are 'covalently closed, single-stranded transcripts comprising many RNA species' that are ubiquitous throughout molecular biology (7). Although circRNAs have been observed in eukaryotic cells for decades, they were mainly perceived as products of splicing errors (8). circRNAs are not easily separated from other RNA species by size or electrophoretic mobility (9), hence they are rarely studied intensively. Recent developments in high-throughput deep sequencing and computational approaches have piqued interest in single-stranded circRNA (10).

In the past few years, considerable efforts have been devoted to circRNAs; it has been revealed that they are closely related to the risk of atherosclerotic vascular disease and neurological disorders, among others (11-13). Furthermore, an increasing number of studies have shown that circRNAs participate in tumorigenesis, metastasis, and other malignant cancer processes (14-18). Memczak et al (19) and Hansen et al (13) reported that circRNAs containing microRNA (miRNA/miR) response elements could interact with miRNAs as competitive endogenous RNAs (ceRNAs) to regulate the expression of target mRNAs. Since then, accumulating circRNA dysregulation was found to be associated with $\mathrm{BC}$ by functioning as ceRNAs $(20,21)$. High-throughput sequencing and transcriptional analysis have been carried out to elucidate the association between circRNAs and BC, revealing considerable circRNA expression in $\mathrm{BC}(22,23)$. Information regarding circRNA expression in different grades of BC remains very limited; therefore, we investigated differences in the circRNA expression profiles of different grades of $\mathrm{BC}$ and normal urothelial cells to identify novel targets for the diagnosis and treatment of BC.

In the present study, we sequenced tissues from different grades of BC and normal controls to define their circRNA expression profiles. We focused on hsa_circ_0137606, which was significantly downregulated in $\mathrm{BC}$, finding that it could suppress BC cell proliferation and metastasis by sponging miR-1231. We aimed to provide potential therapeutic targets for MIBC.

\section{Materials and methods}

Tissue collection. Our study was conducted according to the recommendations of the Declaration of Helsinki and was approved by the Ethics Committee of Nanjing Drum Tower Hospital and the Affiliated Hospital of Nanjing University Medical School. Patients with a history of other cancers, preoperative chemotherapy, or radiotherapy were excluded. Each patient provided written informed consent before tissue samples were collected. A total of 13 patients were employed for tissue collection (age 48-74; 5 males, 8 females), comprising high-grade $\mathrm{BC}$, low-grade $\mathrm{BC}$ and a normal control. All samples were placed in frozen storage tubes with RNAlater
(Thermo Fisher Scientific, Inc.), immediately frozen in liquid nitrogen, and stored at $-80^{\circ} \mathrm{C}$. Three pairs of tissue specimens were randomly chosen for high-throughput sequencing.

RNA extraction. TRIzol ${ }^{\circledR}$ (Thermo Fisher Scientific, Inc.) was used to extract total RNA from the paired cancer and normal control tissues according to the manufacturer's instructions. RNA purity and concentration were checked by A260/A230 (>1.6) and OD A260/A280 (>1.8). Quality and yield were assessed using an Agilent 2100 Bioanalyzer (Agilent Technologies, Inc.) and RNA 6000 Nano Lab Chip Kit (Agilent Technologies, Inc.).

Sequencing process and analysis. Whole transcriptome library preparation and deep sequencing were conducted by Biomarker Technologies Co, Ltd. Total RNA quality and purity were determined using an ultra-micro spectrophotometer (optical density 260 nm, NanoDrop; Thermo Fisher Scientific, Inc.) and circRNA sequencing libraries were constructed according to the manufacturer's recommendations. IlluminaHiSeq 4000 sequencing (Illumina, Inc.) was used to sequence the libraries. FastQC was used to check the raw data. After the data had been filtered, clean circRNA reads were mapped on to the human reference genome (release hg19). Spliced reads per billion mapping was used to quantify circRNA expression levels. DESeq R package was used to identify the significantly dysregulated circRNAs with cut-off criteria: $\mathrm{P}<0.05$ and $\log 2$ fold changel $>1$.

Reverse transcription-quantitative polymerase chain reaction $(R T-q P C R)$. After RNA was isolated from the 13 tissue specimen pairs, cDNA was synthesized using M-MLV reverse transcriptase (Invitrogen; Thermo Fisher Scientific, Inc.) according to the manufacturer's instructions. qPCR (ABI VII7 PCR System, Applied Biosystems; Thermo Fisher Scientific, Inc.) was conducted in a $20 \mu \mathrm{l}$ reaction volume $(10 \mu \mathrm{l} \mathrm{SYBR}$ Green Master Mix, 0.8 $\mu$ PCR Forward Primer (10 $\mu \mathrm{M}), 0.8 \mu \mathrm{l}$ PCR Reverse Primer $(10 \mu \mathrm{M}), 0.4 \mu \mathrm{l}$ ROX, $2 \mu \mathrm{l} \mathrm{cDNA}$, and $6 \mu \mathrm{l}$ nuclease-free water) with the following protocol: Initiation at $95^{\circ} \mathrm{C}$ for $5 \mathrm{~min}$, followed by 40 cycles of $95^{\circ} \mathrm{C}(5 \mathrm{sec})$ and $60^{\circ} \mathrm{C}$ (34 sec). GAPDH was used as a reference and reactions were performed in three independent wells. The $2^{-\Delta \Delta \mathrm{Cq}}$ method (24) was used to calculate relative RNA expression levels. Primers sequences are presented in Table SI.

Bioinformatics analysis. GO and KEGG pathway analyses were performed to explore the biological functions of dysregulated circRNAs. The R package clusterProfiler (25) was used to analyze biological processes, cellular components and molecular functions enriched for circRNA-derived genes. Hypergeometric testing was performed in the enrichment analysis to identify GO entries significantly enriched compared with the whole genome. The $\mathrm{R}$ package clusterProfiler was used for KEGG pathway analysis to explore the biological pathways in which the dysregulated circRNAs participate. Metascape analysis was performed using online tool metascape (www.metascape. org). Protein-protein-interaction (PPI) network was constructed using online tool STRING (https://string-db.org/). Online tool miRTarBase (http://mirtarbase.mbc.nctu.edu.tw/php/index.php) was used to investigate the miRNA-mRNA interactions. 
A

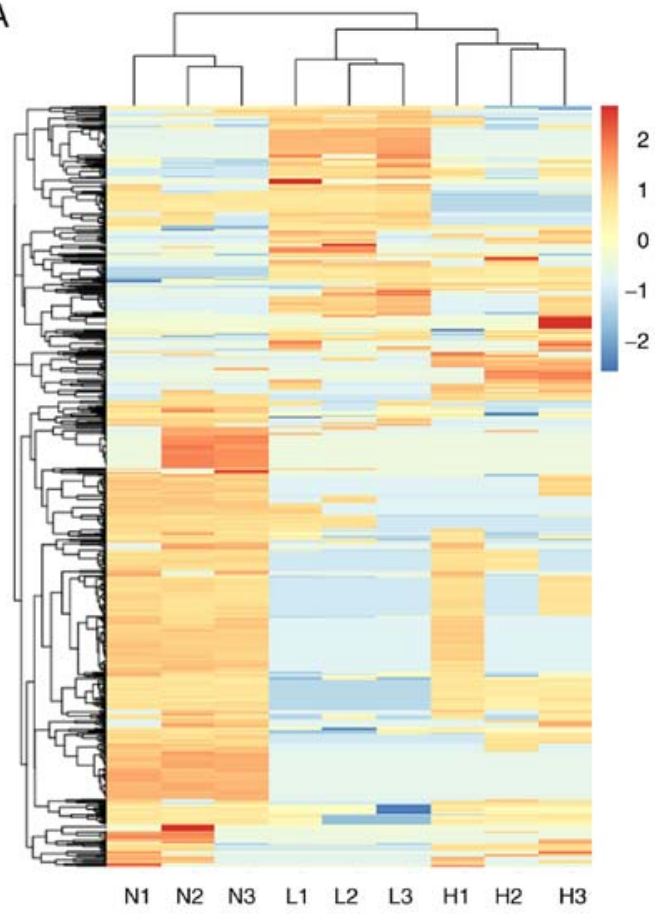

B
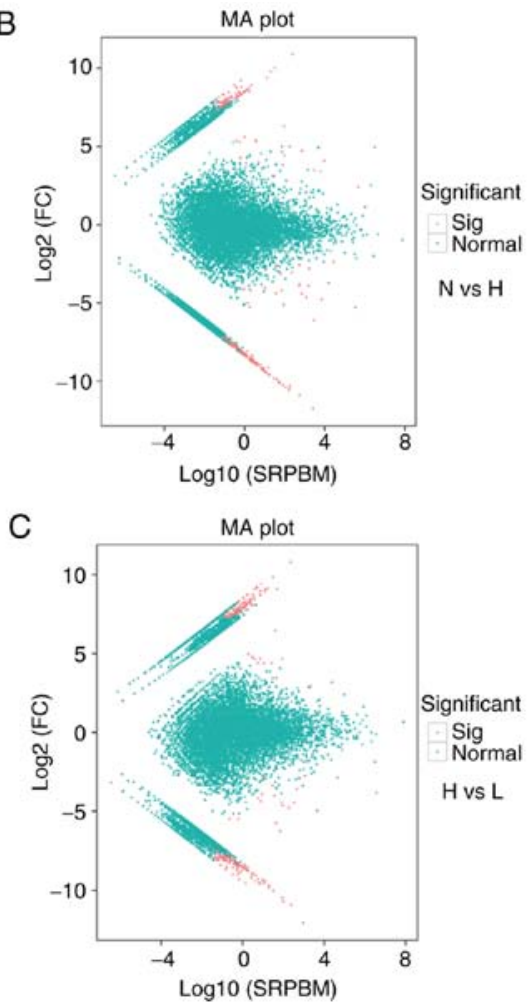

Figure 1. Differentially expressed circRNAs in different grades of bladder cancer and normal urothelium tissues. (A) Heatmap for the expression profiles of circRNAs with significantly different expression in the $\mathrm{H}$ vs. L and $\mathrm{N}$ groups. The key presented the fold change of these dysregulated circRNAs. (B and C) MA plots of circRNAs dysregulated in the two groups. circRNA, circular RNA; H, high-grade bladder cancer; L, low-grade bladder cancer; N, normal tissue.

Cell transfection with small interfering (si)RNA. Hsa circ_0137606 was specifically knocked down using siRNA (5'-GGCAGCTGATGTGCTCATCTT-3') designed by CircInteractome (26) (http://circinteractome.nia.nih.gov) and synthesized (Shanghai GenePharma Co., Ltd.) to target the hsa_circ_0137606 back-splice junction. Scramble siRNA (5'-CCGUGCTGATGTGCTCATCTT-3') was the negative control (NC). T24 cells were transfected with 100 pmol of siRNA using Lipofectamine ${ }^{\circledR} 2000$ (Invitrogen; Thermo Fisher Scientific, Inc.) and harvested after 48 or $72 \mathrm{~h}$ for RT-qPCR or other experiments.

Cell culture. Human BC cell lines T24 and normal Urinary epithelial cells SV-HUC-1 were purchased from American Type Culture Collection. SV-HUC-1 cells were cultured in Dulbecco's Modified Eagles medium (Gibco; Thermo Fisher Scientific, Inc.); T24 cells were cultured with RPMI 1640 (Gibco; Thermo Fisher Scientific, Inc.), containing 10\% fetal bovine serum (Gibco; Thermo Fisher Scientific, Inc.), $100 \mathrm{U} / \mathrm{ml}$ penicillin and $100 \mathrm{mg} / \mathrm{ml}$ streptomycin. All these cell lines were maintained at $37^{\circ} \mathrm{C}$ with $5 \% \mathrm{CO}_{2}$ in a humidified incubator.

Dual-luciferase reporter assay. Hsa_circ_0137606-wild type (WT) and hsa_circ_0137606-mutant (Mut) were constructed using pGL3-Basic luciferase vectors (Promega Corporation) and transfected into BC cells with or without NC or miR-1231 mimics, respectively. After $48 \mathrm{~h}$, a dual-luciferase reporter assay kit (Promega Corporation) was used to determine luciferase activity. Renilla luciferase was used for normalization.
The luciferase activities were measured using a luciferase assay kit (Promega Corporation). Three independent experiments were performed in triplicate.

Cell Counting Kit-8(CCK-8) proliferationassay. Transfected cells were cultured in 96-well plates $(1,000$ cells/well) for 0 , $24,48,72$ or $96 \mathrm{~h}$. According to the manufacturer's protocols, a CCK-8 (Dojindo Molecular Technologies, Inc.) was used to detect cell proliferation. A total of $10 \mu \mathrm{l}$ of CCK-8 solution was added to each well of the 96-well plate and incubated at $37^{\circ} \mathrm{C}$ for $2 \mathrm{~h}$. The absorbance $(450 \mathrm{~nm})$ was measured using a Sunrise Microplate Reader (Tecan Group, Ltd.). Three independent experiments were performed in triplicate.

Transwell assays. A 24-well Transwell chamber (Costar; Corning, Inc.) precoated with or without Matrigel was used to detect cell migratory or invasive abilities, according to the manufacturer's instructions. Cells were cultured in the upper chamber (pre-coated with Matrigel for the invasion assay) with $200 \mathrm{ml}$ serum-free media. The lower chamber was filled with $600 \mathrm{ml}$ of $20 \%$ fetal bovine serum (Gibco; Thermo Fisher Scientific, Inc.) and RPMI 1640 medium. After $48 \mathrm{~h}$, the cells that had migrated to the lower chamber were fixed using formaldehyde (4\%, room temperature, $1 \mathrm{~h}$ ) and stained with $0.1 \%$ crystal violet (room temperature, $20 \mathrm{~min}$ ). The cells were observed and photographed using Axio Observer D1 microscope (magnification, x100 times, Zeiss AG). Three independent experiments were performed in triplicate. 
Table I. Overlapped differential circRNAs in two groups.

\begin{tabular}{|c|c|c|c|c|c|c|}
\hline \multirow[b]{2}{*}{ circRNA } & \multirow[b]{2}{*}{ Position } & \multirow[b]{2}{*}{ Gene symbol } & \multicolumn{2}{|c|}{$\log 2$ cold change } & \multicolumn{2}{|c|}{ P-value } \\
\hline & & & H vs. L & H vs. $\mathrm{N}$ & H vs. L & $\mathrm{H}$ vs. $\mathrm{N}$ \\
\hline hsa_circ_0020840 & chr11:3418346-3418636 & TCONS_12_00004347 & 10.019 & 10.017 & 0.0103 & 0.0079 \\
\hline hsa_circ_0103136 & chr15:24369721-24426402 & TCONS_00023304 & 9.809 & 9.808 & 0.0122 & 0.0094 \\
\hline hsa_circ_0078816 & chr6_cox_hap2:2834337-2836058 & None & 9.398 & 9.388 & 0.0161 & 0.0127 \\
\hline hsa_circ_0030392 & chr13:64608479-64608670 & TCONS_12_00007568 & 8.846 & 8.817 & 0.0065 & 0.0047 \\
\hline hsa_circ_0071818 & chr5:9318483-9380135 & SEMA5A & 8.744 & 8.742 & 0.0301 & 0.0242 \\
\hline hsa_circ_0006329 & chr5:9421788-9437964 & SEMA5A & 8.559 & 8.557 & 0.0359 & 0.0289 \\
\hline hsa_circ_0002099 & chr5:9190378-9238002 & SEMA5A & 8.530 & 8.529 & 0.0370 & 0.0295 \\
\hline hsa_circ_0084606 & chr8:62531536-62566219 & ASPH & 8.521 & 8.511 & 0.0357 & 0.0287 \\
\hline hsa_circ_0136698 & chr8:51415337-51449368 & SNTG1 & 8.487 & 8.485 & 0.0384 & 0.0309 \\
\hline hsa_circ_0099364 & chr12:83250788-83324324 & TMTC2 & 8.472 & 8.470 & 0.0391 & 0.0313 \\
\hline hsa_circ_0071820 & chr5:9337824-9380135 & SEMA5A & 8.472 & 8.470 & 0.0390 & 0.0312 \\
\hline hsa_circ_0081963 & chr7:111127293-111161505 & IMMP2L & 8.451 & 4.722 & 0.0128 & 0.0476 \\
\hline hsa_circ_0063865 & chr22:48081948-48082955 & None & 8.395 & 8.347 & 0.0426 & 0.0359 \\
\hline hsa_circ_0009130 & chr19:1147307-1154401 & SBNO2 & 8.330 & 8.328 & 0.0442 & 0.0360 \\
\hline hsa_circ_0114699 & chr20:13550153-13568017 & TASP1 & 8.292 & 8.274 & 0.0027 & 0.0017 \\
\hline hsa_circ_0007632 & chr20:34312491-34313077 & RBM39 & 8.255 & 8.222 & 0.0145 & 0.0110 \\
\hline hsa_circ_0088274 & chr9:119976636-119977021 & ASTN2 & 8.064 & 8.032 & 0.0072 & 0.0048 \\
\hline hsa_circ_0115417 & chr20:4913100-4951565 & SLC23A2 & 8.052 & 8.023 & 0.0253 & 0.0191 \\
\hline hsa_circ_0140725 & chrY:14493981-14518736 & GYG2P1 & 8.042 & 8.039 & 0.0301 & 0.0221 \\
\hline hsa_circ_0023256 & chr11:68529002-68530229 & CPT1A & 7.956 & 7.913 & 0.0344 & 0.0278 \\
\hline hsa_circ_0001377 & chr3:195605123-195615477 & TNK2 & 7.912 & 7.901 & 0.0375 & 0.0285 \\
\hline hsa_circ_0139039 & chr9:81035195-81038128 & None & 7.891 & 7.872 & 0.0363 & 0.0279 \\
\hline hsa_circ_0007071 & chr5:43122140-43139411 & ZNF131 & 7.850 & 7.812 & 0.0430 & 0.0347 \\
\hline hsa_circ_0082583 & chr7:138209985-138223548 & TRIM24 & 7.846 & 7.818 & 0.0406 & 0.0317 \\
\hline hsa_circ_0006423 & chr1:94140169-94140497 & BCAR3 & 7.840 & 7.814 & 0.0409 & 0.0318 \\
\hline hsa_circ_0128896 & chr5:34922321-34923339 & BRIX1 & 7.495 & 7.482 & 0.0322 & 0.0227 \\
\hline hsa_circ_0124765 & chr3:8977554-9000685 & RAD18 & 7.349 & 7.323 & 0.0442 & 0.0328 \\
\hline hsa_circ_0007766 & chr17:37864573-37866734 & ERBB2 & 3.801 & 4.970 & 0.0124 & 0.0015 \\
\hline hsa_circ_0072654 & chr5:64084777-64100213 & CWC27 & -7.504 & -8.169 & 0.0403 & 0.0017 \\
\hline hsa_circ_0109103 & chr19:13246013-13247219 & NACC1 & -7.520 & -7.334 & 0.0330 & 0.0347 \\
\hline hsa_circ_0004113 & chr3:66293626-66313803 & SLC25A26 & -7.595 & -7.791 & 0.0267 & 0.0336 \\
\hline hsa_circ_0102172 & chr14:56083234-56086030 & KTN1 & -7.677 & -7.347 & 0.0189 & 0.0302 \\
\hline hsa_circ_0008510 & chr9:86279944-86292876 & UBQLN1 & -7.719 & -8.367 & 0.0166 & 0.0010 \\
\hline hsa_circ_0008426 & chr4:129919028-129925031 & SCLT1 & -7.796 & -8.642 & 0.0447 & 0.0004 \\
\hline hsa_circ_0099708 & chr13:100191699-100196249 & TM9SF2 & -7.863 & -8.315 & 0.0112 & 0.0013 \\
\hline hsa_circ_0096402 & chr11:73418460-73429763 & RAB6A & -8.185 & -9.166 & 0.0180 & 0.0001 \\
\hline hsa_circ_0005004 & chr7:44714009-44714867 & OGDH & -8.217 & -8.650 & 0.0028 & 0.0004 \\
\hline hsa_circ_0005746 & chr19:55756487-55757046 & PPP6R1 & -8.332 & -7.899 & 0.0018 & 0.0060 \\
\hline hsa_circ_0125309 & chr4:129042980-129043343 & LARP1B & -8.344 & -7.240 & 0.0024 & 0.0406 \\
\hline hsa_circ_0005429 & chr14:67768105-67770316 & MPP5 & -8.379 & -8.285 & 0.0019 & 0.0015 \\
\hline hsa_circ_0006784 & chr7:151960100-152012423 & MLL3 & -9.080 & -8.338 & 0.0003 & 0.0011 \\
\hline hsa_circ_0001882 & chr9:114148656-114154104 & KIAA0368 & -9.098 & -10.335 & 0.0214 & 0.0005 \\
\hline hsa_circ_0137606 & chr9:107513236-107521452 & NIPSNAP3A & -9.204 & -8.101 & 0.0213 & 0.0494 \\
\hline
\end{tabular}

circRNA, circular RNA; H, high-grade bladder cancer; L, low-grade bladder cancer; N, normal tissue. Bold font indicates the key circRNA of our study.

Statistical analysis. SPSS 21.0 (IBM Corp.) and GraphPad Prism (GraphPad Software, Inc.) was selected for data analysis and plotting. The measured data were presented as the mean \pm standard deviation. Each experiment was 

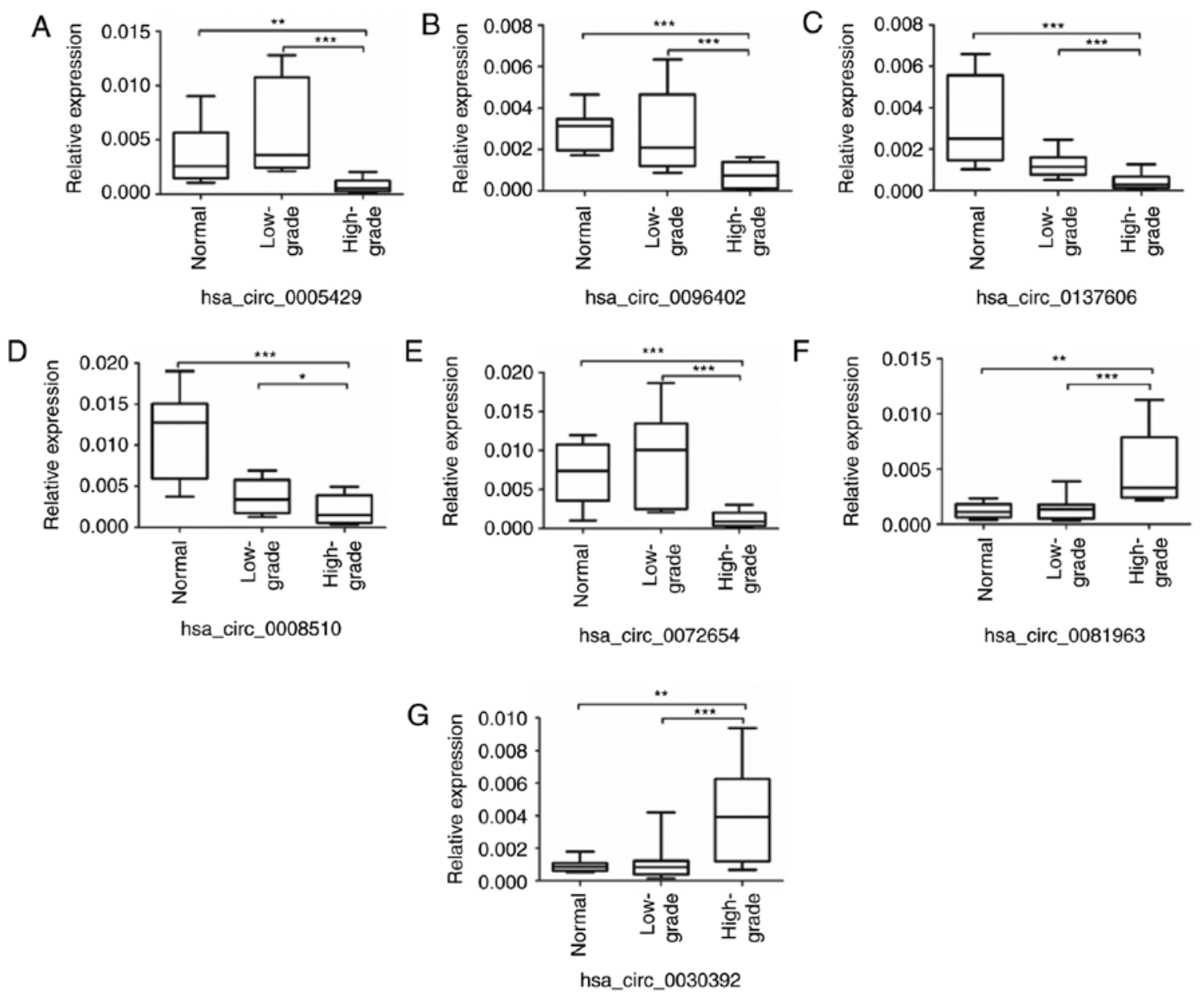

Figure 2. Reverse transcription-quantitative polymerase chain reaction validation of candidate circRNAs in different grades of BC and normal urothelium tissues. (A-E) circRNAs downregulated in high-grade BC compared with low-grade BC and normal urothelium tissues. (F and G) circRNAs upregulated in high-grade $\mathrm{BC}$ compared with low-grade $\mathrm{BC}$ and normal urothelium tissues. Data were shown as the mean \pm standard deviation, ${ }^{*} \mathrm{P}<0.05,{ }^{* *} \mathrm{P}<0.01$ and ${ }_{* * * *} \mathrm{P}<0.001$. BC, bladder cancer; circRNA, circular RNA.

repeated three times. The differences between groups were assessed by a Student's t test and one-way ANOVA. Multiple comparison between the groups was performed using a Student-newman-Keuls post hoc-test. $\mathrm{P}<0.05$ was considered to indicate a statistically significant difference.

\section{Results}

circRNA expression profiles for different grades of $B C$ and normal controls. Heatmaps (Fig. 1A and B) and MA plots (Fig. 1C and D) were used to demonstrate variation in circRNA expression. In high-grade BC tissues, 316 circRNAs were dysregulated compared with NCs (205 upregulated and 111 downregulated) and 244 circRNAs were dysregulated compared with low-grade BC tissues (109 upregulated and 135 downregulated). Further analysis indicated that 42 dysregulated circRNAs overlapped between the two groups (Table I). These differentially expressed circRNAs will be valuable in future studies to reveal the physiopathological mechanisms of $\mathrm{BC}$ progression.

RT-qPCR validation of the differentially expressed circRNAs. To validate the sequencing data, 10 pairs of tissue specimens were collected for RT-qPCR analysis. A total of seven circRNAs were randomly selected for RT-qPCR quantification. As shown in
Fig. 2A-G, the RT-qPCR results were consistent with the expression profiles of our high-throughput sequencing data, in which, hsa_circ_0137606 exhibited a high degree of downregulation.

GO and KEGG pathway analyses. Studies have revealed that circRNAs can exert biological functions by regulating neighboring coding genes (27-29). GO and KEGG pathway analyses were performed on dysregulated circRNAs from the two groups to investigate the mechanisms involved in BC tumorigenesis and progression.

$\mathrm{GO}$ analysis of the $\mathrm{H}$ vs. $\mathrm{N}$ groups demonstrated that the dysregulated circRNAs were enriched for 'extracellular matrix organization' and 'positive GTPase activity' regulation biological processes, 'cell junction' and 'focal adhesion' cellular components, and 'GTPase activity' molecular functions. The top 10 significantly enriched GO terms for the two groups are shown in Figs. 3A-C and S1A-C, whilst their top 10 enriched KEGG pathways are presented in Figs. 3D and S1D. The enrichment network of the dysregulated circRNAs was constructed using metascape (Figs. 4 and S2) (30).

PPI network construction and target gene prediction. To investigate the potential associations between circRNAs and $\mathrm{BC}$ progression, we constructed a protein-protein-interaction 


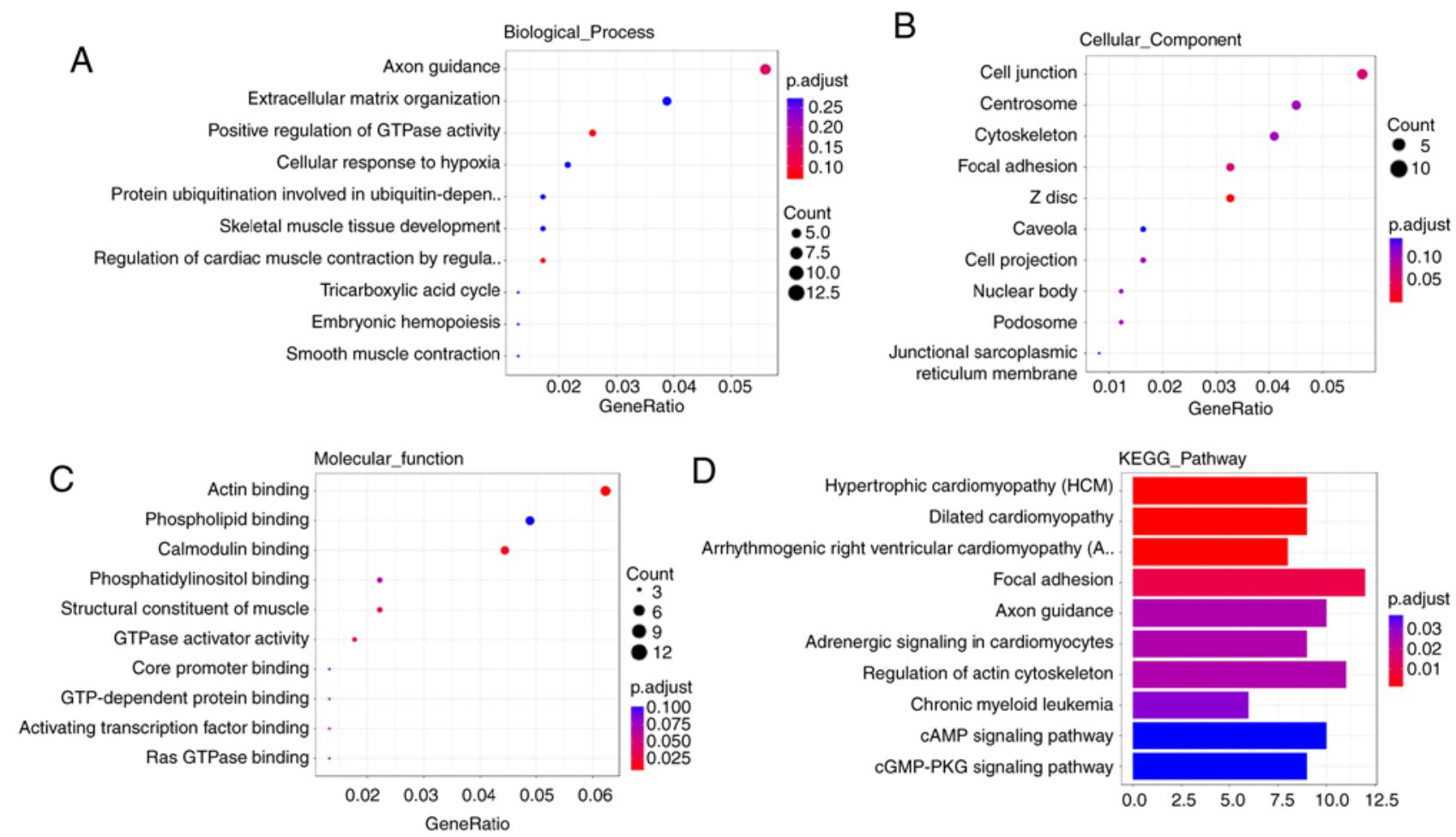

Figure 3. GO and KEGG analyses of the differentially expressed circRNAs in the H vs. N groups. Top $10 \mathrm{GO}$ terms for (A) biological processes, (B) cellular components, and (C) molecular functions for dysregulated circRNAs in the $\mathrm{H}$ vs. N groups. (D) Top 10 KEGG pathways for dysregulated circRNAs in the H vs. N groups. circRNA, circular RNA; GO, Gene Ontology; KEGG, Kyoto Encyclopedia of Genes and Genomes; H, high-grade bladder cancer; N, normal tissue.

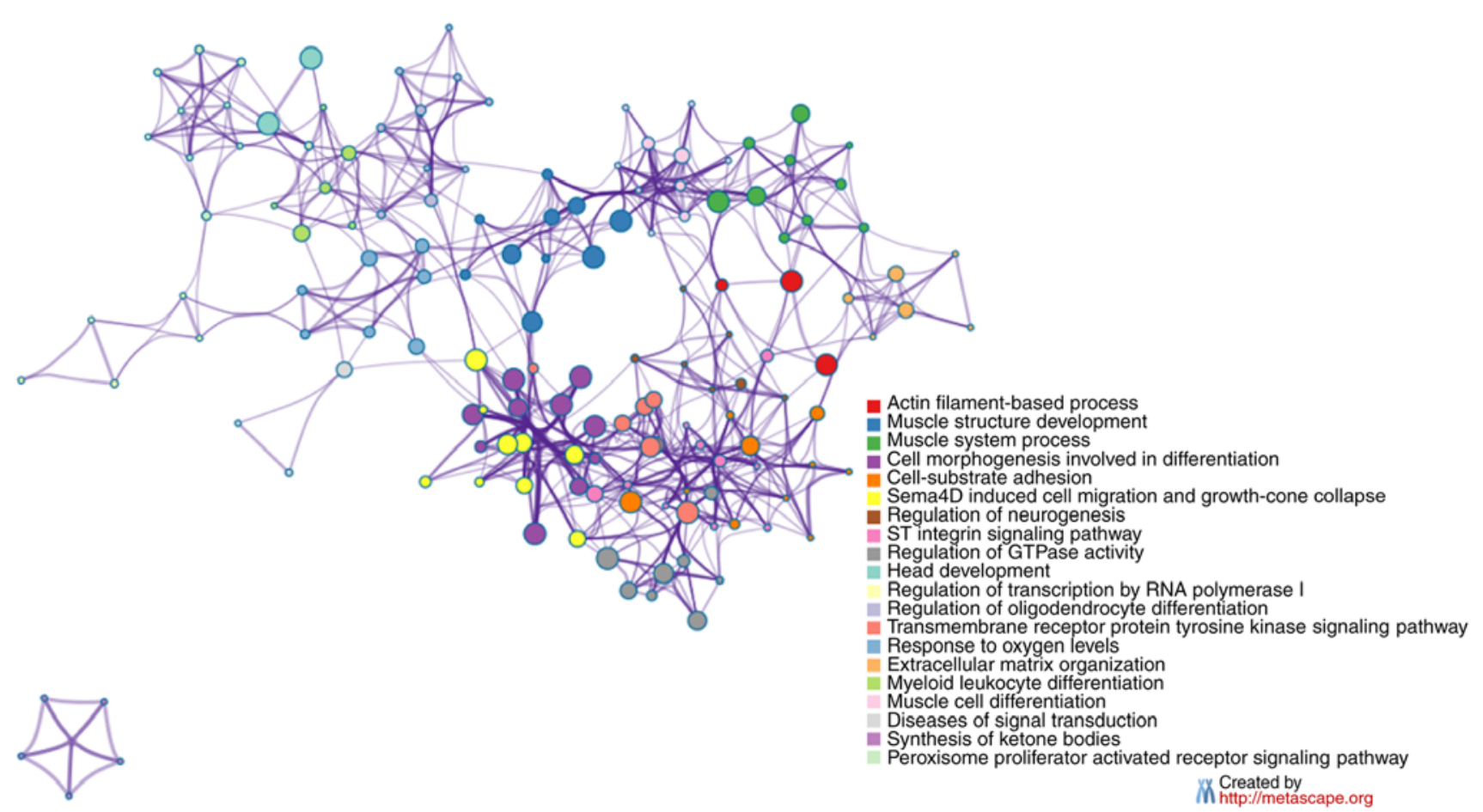

Figure 4. Enrichment network of circRNAs dysregulated in the H vs. N groups. Enrichment network of the circRNAs dysregulated in the H vs. N groups. Nodes represent functions enriched for an annotated ontology term and node size indicates the number of genes that fall into that term. Nodes are clustered into subnetworks that representatively describe the annotations. $\mathrm{P}<0.01$ was the cutoff criteria. circRNA, circular RNA; H, high-grade bladder cancer; $\mathrm{N}$, normal tissue.

(PPI) network based on the circRNA genes dysregulated in both the $\mathrm{H}$ vs. $\mathrm{N}$ and $\mathrm{H}$ vs. L groups. As shown in Fig. S3, receptor tyrosine-protein kinase erbB-2 precursor (ERBB2( exhibited the highest degree of connectivity.
Hsa_circ_0137606 knockdown promotes BC cell proliferation and metastasis. The expression of hsa_circ_0137606 was validated in normal uroepithelial cells (SV-HUC1) and different BC cell lines (Fig. 5A), revealing its significant 

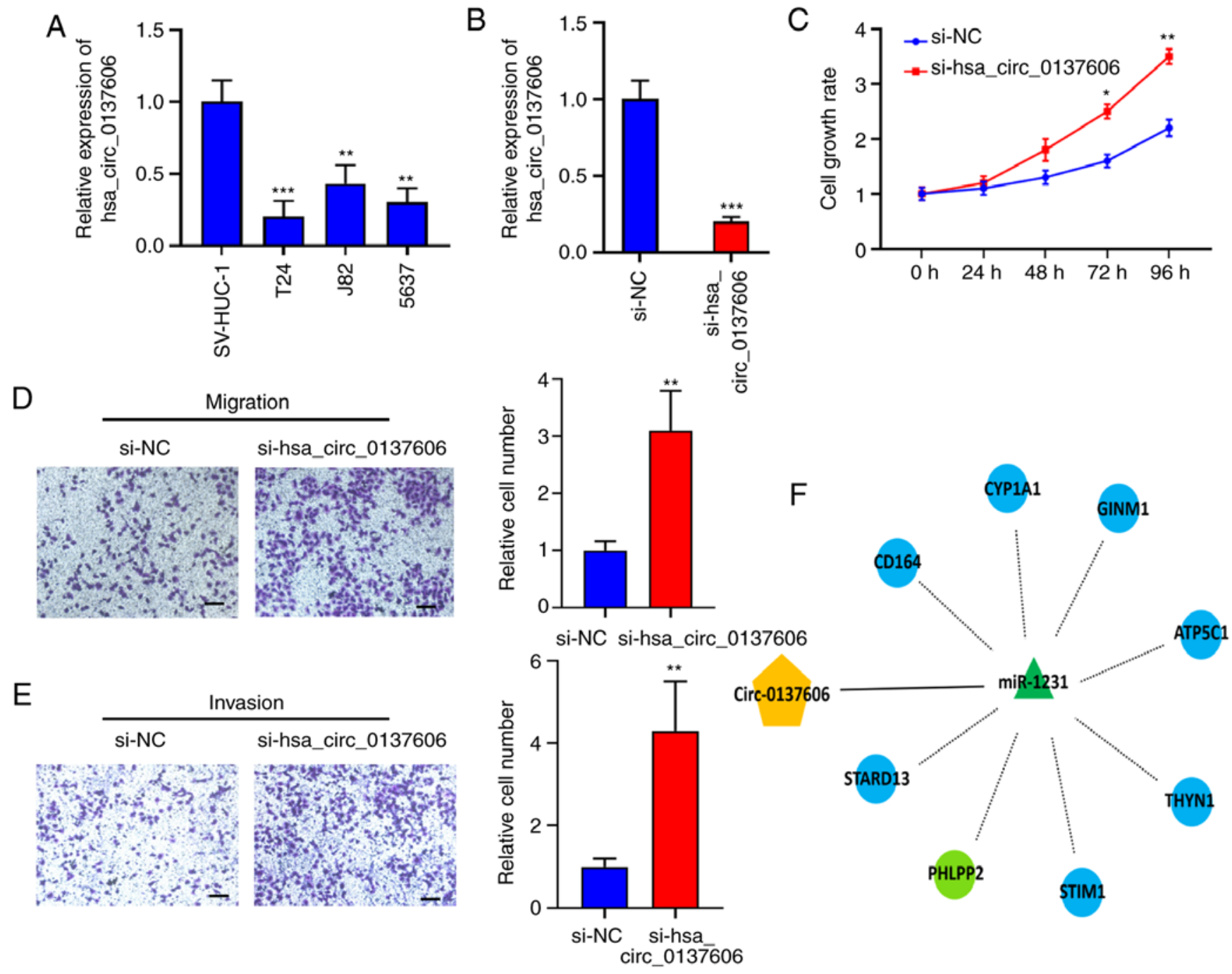

Figure 5. Hsa_circ_0137606 knockdown suppresses BC cell proliferation, migration and invasion. (A) Hsa_circ_0137606 expression in normal uroepithelial cell and different BC cell lines detected with reverse transcription-quantitative polymerase chain reaction. (B) Hsa_circ_0137606 knockdown in T24 cells. (C) Cell Counting Kit-8 assay of proliferation in the knockdown and control groups. (D) Transwell assay of migration in the knockdown and control groups (E) Transwell assay of invasion in the knockdown and control groups. Scale bar, $125 \mu \mathrm{m}$ (F) Candidate target genes predicted by the Circ-interactome of miR-1231 in bladder cancer. Data were shown as the mean \pm standard deviation, ${ }^{*} \mathrm{P}<0.05,{ }^{* *} \mathrm{P}<0.01$ and ${ }^{* * * *} \mathrm{P}<0.001$ vs. si-NC. Hsa, homo sapiens; $\mathrm{NC}$, negative control; si, small interfering RNA; circRNA, circular RNA; ATP5C1, ATP, synthase F1 subunit gamma; CD164, cluster of differentiation 164; CYP1A1, cytochrome P450 1A1; GINM1, glycoprotein integral membrane 1; miR, microRNA; PHLPP2, PH domain and leucine rich repeat protein phosphatase 2; STIM1, stromal interaction molecule 1; STARD13, StAR related lipid transfer domain containing 13; THYN1, thymocyte nuclear protein 1.

downregulation in different $\mathrm{BC}$ cells compared with SV-HUC1 cells. To investigate its biological function in $\mathrm{BC}$, we knocked down hsa_circ_0137606 in T24 cells. RT-qPCR revealed that hsa_circ_0137606 expression was significantly decreased in the hsa_circ_0137606-knockdown group compared with the NC group after transfection (Fig. 5B). We performed a CCK-8 assay to explore the effect of hsa_circ_0137606 on cell proliferation; hsa_circ_0137606 knockdown led to significant increases in $\mathrm{BC}$ cell proliferation compared with the NC group at 72 and $96 \mathrm{~h}$ (Fig. 5C). The Transwell assays revealed that hsa_circ_0137606 knockdown significantly promoted BC cell migration (Fig. 5D) and invasion (Fig. 5E) compared with the NC group. In summary, hsa_circ_0137606 knockdown promoted the proliferation and metastasis of $\mathrm{BC}$ cells in vitro.

Using bioinformatics analysis, we predicted potential target genes that miR-1231 may bind to and regulate during BC progression; the miRNA-mRNA interactions were validated via miRTarBase. As presented in Fig. 5F, the top eight predicted target genes that miR-1231 may regulate during BC progression were identified.

Hsa_circ_0137606 suppresses BC proliferation and metastasis by sponging miR-1231. circRNAs can act as miRNA sponges to regulate cells; therefore, we performed bioinformatics analysis and predicted the miRNAs that hsa_circ_0137606 could act as a sponge for; miR-1248, miR-1263, miR-1298 and miR-1231 were the four most likely miRNAs. To validate this prediction, we determined the expression of the four miRNAs in the hsa_circ_0137606-knockdown and control groups, finding that only miR-1231 was significantly increased in the knockdown group (Fig. 6A). Luciferase assays were performed to further investigate the role of miR-1231, revealing that hsa_circ_0137606 could bind to miR-1231 as its sponge (Fig. 6B and C). Rescue experiments showed that miR-1231 inhibitor treatment in 
A

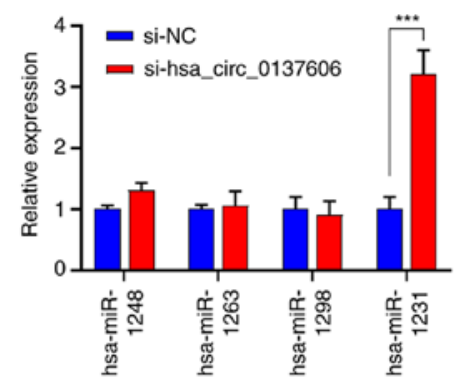

B

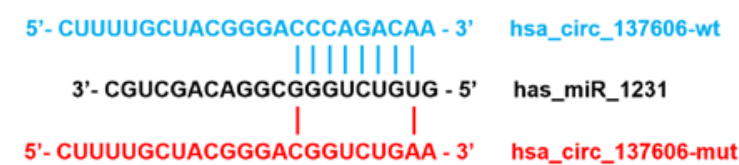

C

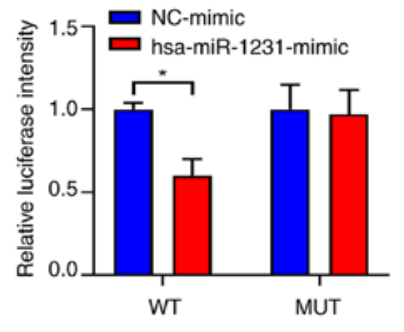

D

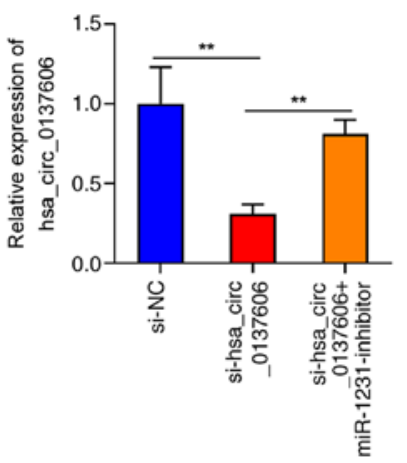

E

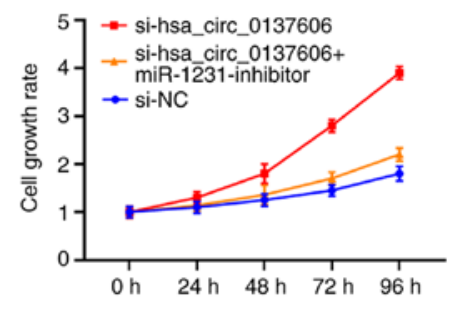

Figure 6. Hsa_circ_0137606 knockdown suppresses BC cell proliferation, migration, and invasion by sponging miR-1231. (A) Expression of different miRNAs in the knockdown and control groups detected by RT-qPCR. ${ }^{* * *} \mathrm{P}<0.001$. (B) miR-1231 and hsa_circ_0137606 binding sites. (C) Luciferase intensity of T24 cells transfected with hsa_circ_0137606 (WT/Mut) and miR-1231 (control/mimics). (D) RT-qPCR reveal the expression level of hsa_circ_0137606 in T24 cells transfected with the indicated plasmids. ${ }^{* *} \mathrm{P}<0.01$. (E) Cell Counting Kit- 8 assay of the proliferation of T24 cells transfected with the indicated plasmids. Hsa, homo sapiens; miR, microRNA; Mut, mutant; NC, negative control; RT-qPCR, reverse transcription-quantitative polymerase chain reaction; si, small interfering RNA; WT, wild type.

hsa_circ_0137606-silenced cells significantly rescued their proliferation (Fig. 6D and E), suggesting that hsa_circ_0137606 suppresses BC proliferation and metastasis by sponging miR-1231.

\section{Discussion}

Since the breakthrough in sequencing technology in 2013 (19), numerous circRNAs have been discovered and many studies have been conducted to investigate their potential functions in disease, particularly cancer (31-34). The mechanism of a few circRNAs in BC tumorigenesis have been revealed $(35,36)$; however, the relationship between circRNAs and the process by which low-grade BC progresses to high-grade BC remains unclear. Therefore, we performed high-throughput sequencing to detect dysregulated circRNAs in different grades of BC and normal controls.

A total of 316 and 244 dysregulated circRNAs were discovered in the $\mathrm{H}$ vs. $\mathrm{N}$ and $\mathrm{H}$ vs. L groups, respectively. Among these, circADAMTS14 has been reported to suppress hepatocellular carcinoma progression by competitively combining with microRNA-572 (37). Moreover, Xu et al (36) found that circPTK2 participates in the proliferation and migration of BC cells. Our further analysis revealed that 42 circRNAs overlapped between the $\mathrm{H}$ vs. $\mathrm{N}$ and $\mathrm{H}$ vs. $\mathrm{L}$ groups.

GO and KEGG pathway analysis were performed to investigate the potential molecular mechanisms of these dysregulated circRNAs. GO analysis revealed that 'GTPase activity regulation' was significantly enriched. GTPases have been strongly implicated in cancer (38); a previous study revealed that dynamin 2 GTPase contributes to the formation of invadopodia, which play an important role in invasive BC cells (12). Liu et al (20) also found that ANXA7 GTPase activity could markedly affect prostate cancer metastasis, indicating that these dysregulated circRNAs could participate in the progression of BC by regulating GTPase activity. Additionally, pathway analysis revealed that 'actin cytoskeleton regulation', 'focal adhesion', and 'cGMP-PKG signaling pathway' were enriched. Peng et al (39) and Ohishi et al (40) found that the actin cytoskeleton serves a crucial role in cancer invasion. The focal adhesion pathway is also involved in cancer cell invasion (41), migration (42), and therapy resistance (23). Consequently, we speculated that the dysregulated circRNAs we detected predominantly participate in $\mathrm{BC}$ progression via such pathways.

To further investigate the association between circRNAs and $\mathrm{BC}$, we constructed a PPI network based on the circRNA gene symbols dysregulated in both the $\mathrm{H}$ vs. $\mathrm{N}$ and $\mathrm{H}$ vs. L groups. We found that $E R B B 2$, which serves a key role in the development of breast cancer (43), had the highest degree of connectivity. Previous studies have revealed that $E R B B 2$ could be involved in BC $(44,45)$, indicating that the differently expressed circRNAs may affect $\mathrm{BC}$ progression by regulating $E R B B 2$.

To validate our results, we performed RT-qPCR on seven circRNAs in independent tissues; the novel circRNA, hsa_circ_0137606, was selected for further analysis. RT-qPCR was performed on normal uroepithelial cells and different BC cell lines to validate its expression, finding it to be significantly downregulated in BC cells (particularly T24 cells). Functional in vitro experiments demonstrated that hsa_circ_0137606 could suppress BC cell proliferation, migration and invasion. A previous study (46) indicated that circRNAs containing 
miRNA-binding sites could act as cellular miRNA sponges. By binding miRNA, they could prevent its inhibitory effect on target genes and thus indirectly regulate their expression. By using bioinformatics and RT-qPCR analyses, we predicted that hsa_circ_0137606 could act as a sponge for miR-1231, with luciferase reporter assays further validating this finding. Rescue experiments showed that inhibiting miR-1231 significantly rescued hsa_circ_0137606 knockdown-induced proliferation, whilst previous studies have shown that miR-1231 has a role in multiple malignant tumors $(47,48)$. Furthermore, we used miRTarBase to predict the eight most likely target genes of miR-1231, one of which was $\mathrm{PH}$ domain and leucine rich repeat protein phosphatase 2 (PHLPP2I) which has been shown to be involved in $\mathrm{BC}$ progression as an miRNA target gene (49,50); thus, PHLPP2 may be a promising target of miR-1231 in BC. However, some limitations should be mentioned in this study. First, the number of samples for sequencing was rather low, more samples should be employed for future detection to verify our results. Secondly, when the expression of hsa_circ_0137606 was explored in different BC cell lines, we did not select a non-bladder cell as control group. Non-bladder control cells could improve the reliability of the results. In conclusion, we used high-throughput sequencing to identify aberrantly expressed circRNAs in different grades of BC. Using bioinformatics analysis, we found that these dysregulated circRNAs could synergistically contribute towards BC progression. Furthermore, we revealed that hsa_circ_0137606, which is significantly downregulated in BC, could suppress $\mathrm{BC}$ proliferation and metastasis by sponging miR-1231. This study suggests that hsa_circ_0137606 could be an effective therapeutic target for $\mathrm{BC}$.

\section{Acknowledgements}

Not applicable.

\section{Funding}

The present study was financially supported by the National Natural Science Foundation of China (grant nos. 81772712 and 81702569) and the Natural Science Foundation of Jiangsu Province (grant no. BK20170151).

\section{Availability of data and materials}

The datasets used and/or analyzed during the current study are available from the corresponding author on reasonable request.

\section{Authors' contributions}

WL made substantial contributions to the design of the present study; YC and JZ acquired the data. ZS and WM performed the experiments. LY analyzed and interpreted the data; KX and $\mathrm{XY}$ were involved in drafting the manuscript and revising it critically for important intellectual content.

\section{Ethics approval and consent to participate}

The present study was approved by the Ethics Committee of Nanjing Drum Tower Hospital and the Affiliated Hospital of
Nanjing University Medical School. Written informed consent was obtained from patients.

\section{Patient consent for publication}

Not applicable.

\section{Competing interests}

The authors declare that they have no competing interests.

\section{References}

1. Antoni S, Ferlay J, Soerjomataram I, Znaor A, Jemal A and Bray F: Bladder cancer incidence and mortality: A global overview and recent trends. Eur Urol 71: 96-108, 2017.

2. Cumberbatch MGK, Jubber I, Black PC, Esperto F, Figueroa JD, Kamat AM, Kiemeney L, Lotan Y, Pang K, Silverman DT, et al: Epidemiology of bladder cancer: A systematic review and contemporary update of risk factors in 2018. Eur Urol 74: 784-795, 2018.

3. Kamat AM, Hahn NM, Efstathiou JA, Lerner SP, Malmström PU, Choi W, Guo CC, Lotan Y and Kassouf W: Bladder cancer. Lancet 388: 2796-2810, 2016.

4. Soukup V, Čapoun O, Cohen D, Hernández V, Babjuk M, Burger M, Compérat E, Gontero P, Lam T, MacLennan S, et al: Prognostic performance and reproducibility of the 1973 and $2004 / 2016$ world health organization grading classification systems in non-muscle-invasive bladder cancer: A European association of urology non-muscle invasive bladder cancer guidelines panel systematic review. Eur Urol 72: 801-813, 2017.

5. Funt SA and Rosenberg JE: Systemic, perioperative management of muscle-invasive bladder cancer and future horizons. Nat Rev Clin Oncol 14: 221-234, 2017.

6. Sargos P, Baumann BC, Eapen L, Christodouleas J, Bahl A, Murthy V, Efstathiou J, Fonteyne V, Ballas L, Zaghloul M, et al: Risk factors for loco-regional recurrence after radical cystectomy of muscle-invasive bladder cancer: A systematic-review and framework for adjuvant radiotherapy. Cancer Treat Rev 70: 88-97, 2018.

7. Chen W, Zheng R, Baade PD, Zhang S, Zeng H, Bray F, Jemal A, Yu XQ and He J: Cancer statistics in China, 2015. CA Cancer J Clin 66: 115-132, 2016.

8. Vicens Q and Westhof E: Biogenesis of circular RNAs. Cell 159: 13-14, 2014

9. Jeck WR and Sharpless NE: Detecting and characterizing circular RNAs. Nat Biotechnol 32: 453-461, 2014.

10. Li X, Yang L and Chen LL: The biogenesis, functions, and challenges of circular RNAs. Mol Cell 71: 428-442, 2018.

11. Holdt LM, Stahringer A, Sass K, Pichler G, Kulak NA, Wilfert W, Kohlmaier A, Herbst A, Northoff BH, Nicolaou A, et al: Circular non-coding RNA ANRIL modulates ribosomal RNA maturation and atherosclerosis in humans. Nat Commun 7: 12429, 2016.

12. Chen F, Chen X, Yang D, Che X, Wang J, Li X, Zhang Z, Wang Q, Zheng W, Wang L, et al: Isoquercitrin inhibits bladder cancer progression in vivo and in vitro by regulating the PI3K/Akt and PKC signaling pathways. Oncol Rep 36: 165-172, 2016.

13. Hansen TB, Jensen TI, Clausen BH, Bramsen JB, Finsen B, Damgaard CK and Kjems J: Natural RNA circles function as efficient microRNA sponges. Nature 495: 384-388, 2013.

14. Han D, Li J, Wang H, Su X, Hou J, Gu Y, Qian C, Lin Y, Liu X, Huang M, et al: Circular RNA circMTO1 acts as the sponge of microRNA-9 to suppress hepatocellular carcinoma progression. Hepatology 66: 1151-1164, 2017.

15. Yu J, Xu QG, Wang ZG, Yang Y, Zhang L, Ma JZ, Sun SH, Yang F and Zhou WP: Circular RNA cSMARCA5 inhibits growth and metastasis in hepatocellular carcinoma. J Hepatol 68: 1214-1227, 2018.

16. Chen X, Chen RX, Wei WS, Li YH, Feng ZH, Tan L, Chen JW, Yuan GJ, Chen SL, Guo SJ, et al: PRMT5 circular RNA promotes metastasis of urothelial carcinoma of the bladder through sponging miR-30c to induce epithelial-mesenchymal transition. Clin Cancer Res 24: 6319-6330, 2018.

17. Chen Y, Yang F, Fang E, Xiao W, Mei H, Li H, Li D, Song H, Wang J, Hong M, et al: Circular RNA circAGO2 drives cancer progression through facilitating HuR-repressed functions of AGO2-miRNA complexes. Cell Death Differ 26: 1346-1364, 2019. 
18. Weng W, Wei Q, Toden S, Yoshida K, Nagasaka T, Fujiwara T, Cai S, Qin H, Ma Y and Goel A: Circular RNA ciRS-7-A promising prognostic biomarker and a potential therapeutic target in colorectal cancer. Clin Cancer Res 23: 3918-3928, 2017.

19. Memczak S, Jens M, Elefsinioti A, Torti F, Krueger J, Rybak A, Maier L, Mackowiak SD, Gregersen LH, Munschauer M, et al: Circular RNAs are a large class of animal RNAs with regulatory potency. Nature 495: 333-338, 2013.

20. Liu S, Li X, Lin Z, Su L, Yan S, Zhao B and Miao J: SEC-induced activation of ANXA7 GTPase suppresses prostate cancer metastasis. Cancer Lett 416: 11-23, 2018.

21. Li Y, Zheng F, Xiao X, Xie F, Tao D, Huang C, Liu D, Wang M, Wang L, Zeng F and Jiang G: CircHIPK3 sponges miR-558 to suppress heparanase expression in bladder cancer cells. EMBO Rep 18: 1646-1659, 2017.

22. Li M, Liu Y, Zhang X, Liu J and Wang P: Transcriptomic analysis of high-throughput sequencing about circRNA, lncRNA and mRNA in bladder cancer. Gene 677: 189-197, 2018.

23. Eke I and Cordes N: Focal adhesion signaling and therapy resistance in cancer. Semin Cancer Biol 31: 65-75, 2015.

24. Livak KJ and Schmittgen TD: Analysis of relative gene expression data using real-time quantitative PCR and the 2(-Delta Delta C(T)) method. Methods 25: 402-408, 2001.

25. Yu G, Wang LG, Han Y and He QY: clusterProfiler: An R package for comparing biological themes among gene clusters. OMICS 16: 284-287, 2012.

26. Chou CH, Shrestha S, Yang CD, Chang NW, Lin YL, Liao KW, Huang WC, Sun TH, Tu SJ, Lee WH, et al: miRTarBase update 2018: A resource for experimentally validated microRNA-target interactions. Nucleic Acids Res 46 (D1): D296-D302, 2018.

27. Yao Y, Chen X, Yang H, Chen W, Qian Y, Yan Z, Liao T, Yao W, Wu W, Yu T, et al: Hsa_circ_0058124 promotes papillary thyroid cancer tumorigenesis and invasiveness through the NOTCH3/GATAD2A axis. J Exp Clin Cancer Res 38: 318, 2019.

28. Wu K, Liao X, Gong Y, He J, Zhou JK, Tan S, Pu W, Huang C, Wei YQ and Peng Y: Circular RNA F-circSR derived from SLC34A2-ROS1 fusion gene promotes cell migration in non-small cell lung cancer. Mol Cancer 18: 98, 2019.

29. Wang L, Long H, Zheng Q, Bo X, Xiao X and Li B: Circular RNA circRHOT1 promotes hepatocellular carcinoma progression by initiation of NR2F6 expression. Mol Cancer 18: 119, 2019.

30. Soonthornvacharin S, Rodriguez-Frandsen A, Zhou Y, Galvez F, Huffmaster NJ, Tripathi S, Balasubramaniam VR, Inoue A, de Castro E, Moulton H, et al: Systems-based analysis of RIG-I-dependent signalling identifies KHSRP as an inhibitor of RIG-I receptor activation. Nat Microbiol 2: 17022, 2017.

31. Kristensen LS, Hansen TB, Veno MT and Kjems J: Circular RNAs in cancer: Opportunities and challenges in the field. Oncogene 37: 555-565, 2018.

32. Liang WC, Wong CW, Liang PP, Shi M, Cao Y, Rao ST, Tsui SK, Waye MM, Zhang Q, Fu WM and Zhang JF: Translation of the circular RNA circ $\beta$-catenin promotes liver cancer cell growth through activation of the Wnt pathway. Genome Biol 20: 84 , 2019.

33. Braicu C, Zimta AA, Gulei D, Olariu A and Berindan-Neagoe I: Comprehensive analysis of circular RNAs in pathological states: Biogenesis, cellular regulation, and therapeutic relevance. Cell Mol Life Sci 76: 1559-1577, 2019.

34. Vo JN, Cieslik M, Zhang Y, Shukla S, Xiao L, Zhang Y, Wu YM, Dhanasekaran SM, Engelke CG, Cao X, et al: The landscape of circular RNA in cancer. Cell 176: 869-881.e13, 2019.

35. Xie F, Li Y, Wang M, Huang C, Tao D, Zheng F, Zhang H, Zeng F, Xiao X and Jiang G: Circular RNA BCRC-3 suppresses bladder cancer proliferation through miR-182-5p/p27 axis. Mol Cancer 17: 144, 2018.

36. Xu ZQ, Yang MG, Liu HJ and Su CQ: Circular RNA hsa circ_0003221 (circPTK2) promotes the proliferation and migration of bladder cancer cells. J Cell Biochem 119: 3317-3325, 2018.
37. Song C, Li D, Liu H, Sun H, Liu Z, Zhang L and Hu Y: The competing endogenous circular RNA ADAMTS14 suppressed hepatocellular carcinoma progression through regulating microRNA-572/regulator of calcineurin 1. J Cell Physiol 234: 2460-2470, 2019.

38. Subramani D and Alahari SK: Integrin-mediated function of Rab GTPases in cancer progression. Mol Cancer 9: 312, 2010.

39. Peng JM, Bera R, Chiou CY, Yu MC, Chen TC, Chen CW, Wang TR, Chiang WL, Chai SP, Wei Y, et al: Actin cytoskeleton remodeling drives epithelial-mesenchymal transition for hepatoma invasion and metastasis in mice. Hepatology 67: 2226-2243, 2018.

40. Ohishi T, Yoshida H, Katori M, Migita T, Muramatsu Y, Miyake M, Ishikawa Y, Saiura A, Iemura SI, Natsume T and Seimiya H: Tankyrase-binding protein TNKS1BP1 regulates actin cytoskeleton rearrangement and cancer cell invasion. Cancer Res 77: 2328-2338, 2017.

41. Schlienger S, Ramirez RA and Claing A: ARF1 regulates adhesion of MDA-MB-231 invasive breast cancer cells through formation of focal adhesions. Cell Signal 27: 403-415, 2015.

42. Meng F, Saxena S, Liu Y, Joshi B, Wong TH, Shankar J, Foster LJ, Bernatchez P and Nabi IR: The phospho-caveolin-1 scaffolding domain dampens force fluctuations in focal adhesions and promotes cancer cell migration. Mol Biol Cell 28: 2190-2201, 2017.

43. Petry IB, Fieber E, Schmidt M, Gehrmann M, Gebhard S, Hermes M, Schormann W, Selinski S, Freis E, Schwender H, et al: ERBB2 induces an antiapoptotic expression pattern of Bcl-2 family members in node-negative breast cancer. Clin Cancer Res 16: 451-460, 2010.

44. Junttila TT, Laato M, Vahlberg T, Söderström KO, Visakorpi T, Isola $J$ and Elenius $K$ : Identification of patients with transitional cell carcinoma of the bladder overexpressing ErbB2, ErbB3, or specific ErbB4 isoforms: Real-time reverse transcription-PCR analysis in estimation of ErbB receptor status from cancer patients. Clin Cancer Res 9: 5346-5357, 2003.

45. Groenendijk FH, de Jong J, Fransen van de Putte EE, Michaut M, Schlicker A, Peters D, Velds A, Nieuwland M, van den Heuvel MM, Kerkhoven RM, et al: ERBB2 mutations characterize a subgroup of muscle-invasive bladder cancers with excellent response to neoadjuvant chemotherapy. Eur Urol 69: 384-388, 2016.

46. Liu G, Huang K, Jie Z, Wu Y, Chen J, Chen Z, Fang X and Shen S: CircFAT1 sponges miR-375 to promote the expression of Yes-associated protein 1 in osteosarcoma cells. Mol Cancer 17: 170, 2018.

47. Zhang J, Zhang J, Qiu W, Zhang J, Li Y, Kong E, Lu A, Xu J and $\mathrm{Lu} \mathrm{X:} \mathrm{MicroRNA-1231} \mathrm{exerts} \mathrm{a} \mathrm{tumor} \mathrm{suppressor} \mathrm{role}$ through regulating the EGFR/PI3K/AKT axis in glioma. J Neurooncol 139: 547-562, 2018.

48. Zhou C, Yu Q, Chen L, Wang J, Zheng S and Zhang J: A miR-1231 binding site polymorphism in the 3'UTR of IFNAR1 is associated with hepatocellular carcinoma susceptibility. Gene 507: 95-98, 2012.

49. Mao XP, Zhang LS, Huang B, Zhou SY, Liao J, Chen LW, Qiu SP and Chen JX: Mir-135a enhances cellular proliferation through post-transcriptionally regulating PHLPP2 and FOXO1 in human bladder cancer. J Transl Med 13: 86, 2015.

50. Huang C, Liao X, Jin H, Xie F, Zheng F, Li J, Zhou C, Jiang G, Wu XR and Huang C: MEG3, as a competing endogenous RNA, binds with miR-27a to promote PHLPP2 protein translation and impairs bladder cancer invasion. Mol Ther Nucleic Acids 16: 51-62, 2019.

This work is licensed under a Creative Commons Attribution-NonCommercial-NoDerivatives 4.0 International (CC BY-NC-ND 4.0) License. 